\title{
Actitudes sexuales en adolescentes estudiantes universitarios
}

ISSN 1794-9831

E-ISSN 2322-7028

Vol. 12 No. 1

Ene - Jun 2015

Cúcuta, Colombia

\author{
Gloria Esperanza Zambrano Plata* \\ Jessica Paola Toscano Moros** \\ Jennifer Gil Marles ${ }^{* * *}$
}

Recibido:

24 de Febrero

de 2015

Aprobado:

11 de Mayo de 2015

\footnotetext{
*Enfermera

Doctora en

Educación.

Docente.

Universidad

Francisco de

Paula Santander.

Correo electrónico:

gloriae91@gmail.

com

** Enfermera

IPS Unipamplona.

Correo electrónico:

jessica toscano@

hotmail.com

*** Enfermera

Clínica San José

de Cúcuta. Correo

electrónico:

jeny9046@

hotmail.com

**** Descriptores

en Ciencias de la

Salud (DeCS), en

la página http://

decs.bvs.br/E/

homepagee.htm

de la Biblioteca

virtual en salud del

proyecto BIREME,

de la Organización

Mundial de la

Salud y de la

Organización

Panamericana de

Salud.
} 


\section{Sexual attitudes among university students}

\section{ABSTRACT}

Objective: to describe the sexual attitudes of adolescents enrolled in day programs in a university in Cucuta, Colombia, in the first half of 2014. Materials and Methods: quantitative, descriptive approach. The sample consisted of 375 adolescents university students. The selection of subjects was performed using a multistage stratified sampling. The information was collected through the Sexual Attitudes instrument. Results: participants were on average 19 years old, predominantly male genre, Catholic religion, with an origin in nuclear families and heterosexual orientation. Liberal sexual attitudes were $68 \%$ of surveyed adolescents. This percentage increased about the attitude to face sexual relationships, where $75 \%$ maintained a liberal stance percentage, while analyzing the attitude towards different ways of practicing sex was found $53 \%$ with conservative and the percentage increased when it was analyzed the attitude to the free expression of sexuality with $60 \%$ of students with conservative stance. Regarding the relationship of some variables with liberal attitudes towards sexuality, it was found a statistically significant relationship between this and the fact of being a man, being older than 19 years old, belonging to higher semesters, being agnostic or atheist, going or not to the church and being bisexual. Conclusions: the sexual liberalism in the attitudes of university adolescents is more discursive than practice, because this posture changes according to the context.

KEYWORDS: adolescent, attitude, sexuality 


\section{As atitudes sexuais entre universitários}

Objetivo: descrever as atitudes sexuais de adolescentes matriculados em programas de dia enfrentar uma universidade no Cúcuta, Colombia, no primeiro semestre de 2014. Materiais e Métodos: abordagem quantitativa, descritivo. A amostra foi composta de 375 adolescentes universitarios. A seleção dos sujeitos foi realizada utilizando uma amostragem estratificada em múltiplos estágios. A informação foi recolhida através das Atitudes Sexuais de instrumentos. Resultados: os participantes tinham em média 19 anos, predominantemente do sexo masculino, religião católica, de origem das famílias nucleares e orientação heterossexual. Atitudes sexuais liberais eram $68 \%$ dos entrevistados. Este aumento em relação a atitude em relação ao sexo, onde $75 \%$ mantiveram um percentual liberal postura, ao analisar a atitude em relação a diferentes formas de praticar sexo foi encontrado 53\% com conservadora e o aumento percentual quando analisados atitude para a livre expressão da sexualidade com $60 \%$ de alunos com postura conservadora. Quanto à relação de algumas variáveis com atitudes liberais em relação a sexualidade, a relação estatisticamente significativa entre este homem e ser encontrado, não inferior a 19 anos, pertencentes a semestres mais elevadas, ser agnóstico ou ateu, pouco ou participar Nunca ao templo e ser bissexual. Conclusões: o liberalismo sexual nas atitudes dos adolescentes da faculdade é mais discursiva e eu praticar esta postura, muda de acordo com o contexto.

PALAVRAS-CHAVE: adolescentes, atitude, sexualidade. 


\section{INTRODUCCIÓN}

Ene - Jun 2015 Cúcuta, Colombia
$\mathrm{L}$ a adolescencia es un periodo de cambios no solamente a nivel fisiológico sino también a nivel psicológico y socio-cultural. En esta etapa se definen aspectos importantes como la imagen corporal, la identidad, la propia personalidad y otras tantas cuestiones de la vida, entre las que se encuentra una muy importante: la sexualidad. Este aspecto se relaciona no sólo con la orientación sexual sino también con las actitudes que adopta el adolescente frente al ejercicio de su sexualidad, las cuales son influenciadas por el tipo de educación, la crianza y los valores recibidos durante su crecimiento, además del contexto en el que se desenvuelven, amigos, medios de comunicación, etc. (1-2).

La Organización Mundial de la Salud (OMS) la define como el período de vida en el cual el individuo adquiere la capacidad reproductiva, transita los patrones psicológicos de la niñez a la adultez y consolida la independencia socioeconómica; fija sus límites entre los 10 y 20 años (3).

La adolescencia constituye un período de la vida donde ocurren una serie de cambios con rapidez vertiginosa, los cuales se reflejan en la esfera anatomofisiológica, sicológica, social y cultural, que provocan que sea cada vez más necesario dedicarle mayor atención. Las decisiones que tome un adolescente durante esta etapa, en materia de sexualidad, pueden llevarlo a situaciones de riesgo que pueden alterar su estado de salud y afectar, en última instancia, su calidad de vida.

Durante la adolescencia se consolidan actitudes hacia la sexualidad, las cuales influyen significativamente en la forma en que los individuos viven y expresan este aspecto vital a lo largo de su ciclo de desarrollo. Las actitudes son predisposiciones a responder hacia algo de determinada forma, ya sea mediante reacciones favorables o desfavorables. Estas actitudes las integran: opiniones, sentimientos, creencias, modas o tendencias que se forman durante la vida a través de experiencias y vivencias significativas que obtienen las personas al interactuar con su entorno (2).

Las actitudes hacia la sexualidad humana han estado siempre relacionadas con la moral imperante en la sociedad de la época (4). La actitud de los adolescentes hacia la sexualidad, la contracepción y el contagio de enfermedades de transmisión sexual varía según el nivel cultural, las creencias religiosas, las relaciones afectivas con los padres y la edad de los adolescentes.

Según Hatano (5), en las últimas décadas se ha observado una tendencia a una mayor liberalización de los comportamientos y actitudes sexuales en el mundo occidental. Esta tendencia, constatada en numerosos estudios y que en algunos países ha comenzado a invertirse, se manifiesta de forma especial entre los adolescentes.

En Colombia, la mayoría de los estudiantes universitarios se encuentra en la adolescencia. Esto se observa en una universidad pública de Cúcuta Colombia, en donde el $77,13 \%$ de los estudiantes que inician su formación superior se encuentran en el rango de edad de 15 a 19 años, etapa crítica del ciclo vital para la definición de sus actitudes y su identidad sexual (6).

El cambio de contexto de un sistema donde contaban con la supervisión y apoyo continuo de los maestros a un ambiente de total libertad, donde la responsabilidad de su ser recae sobre ellos mismos, sumado a la coerción en la convivencia con sus pares y la búsqueda de independencia e individualidad en sus familias, expone al adolescente a situaciones de riesgo en el desarrollo de su sexualidad.

Lasactitudessexualesdeterminanloscomportamientos sexuales, algunos de ellos de riesgo, que ubican a la adolescencia como una etapa de alta vulnerabilidad para la salud sexual y reproductiva. Esto se evidencia en las estadísticas mundiales, que reportaron para el 2008 la cifra de 33.4 millones de personas infectadas por el virus de inmunodeficiencia humana (VIH) (3). En Colombia se reportaron aproximadamente 57.489 casos, de los cuales el 58\% corresponde a adolescentes y adultos jóvenes de los 15 a los 34 años, mientras que en Norte de Santander, para el año 2010, la tasa de incidencia de VIH fue de 9.4 por cada 100.000 habitantes, con un reporte de 122 casos, de los cuales 99 pertenecían al municipio de Cúcuta (7).

Por otra parte, la encuesta de demografía y salud de 2010 revela una tasa general de fecundidad de 84 nacimientos por 1000 mujeres en edad de 15 a 19 años, con 73 nacimientos en zona urbana (8). En Norte de Santander, según datos del Departamento Nacional De Estadística (DANE) y el Instituto Departamental de Salud, para 2010 la cifra de embarazos en 
adolescentes de 10 a 19 años de edad es de 9.570 , con una tasa de 72,67 adolescentes embarazadas por cada 1000 y un reporte total para el departamento de 3.814 abortos en la población de 15 a 44 años de edad (8).

Por esta razón, surge el interés de desarrollar la investigación que sustenta este artículo, con el propósito describir las actitudes sexuales de los adolescentes matriculados en los programas presenciales diurnos en una universidad de la ciudad de Cúcuta (Colombia).

\section{MATERIALES Y MÉTODOS}

Para el logro de los objetivos propuestos se realizó un estudio con enfoque cuantitativo, el cual permitió el análisis de una realidad observable y medible. El método utilizado en la presente investigación es descriptivo, de corte transversal, que tiene como objetivo indagar la incidencia de las Actitudes Sexuales (AS) y explicar sus relaciones con las demás variables dentro de un enfoque cuantitativo.

La población de estudio está compuesta por 15.244 estudiantes activos matriculados en el primer semestre de 2014 en la modalidad de pregrado presencial diurno de una universidad en la ciudad de Cúcuta, Colombia.

Se calculó una muestra de 375 estudiantes, con un nivel de confianza de $95 \%$, un margen de error de $5 \%$ y una probabilidad de ocurrencia del evento de $50 \%$. Se realizó un muestreo polietápico estratificado, proporcional por facultad (ingeniería, salud, empresariales, educación y agrarias). La elección de los sujetos se realizó por cuotas, procedimiento también denominado muestreo accidental.

Para la recolección de la información se aplicó el cuestionario Actitudes Sexuales (AS) diseñado por García (9), el cual presenta una alta consistencia interna, con un Alfa de Cronbach de 0,83; los autores autorizaron su utilización para la presente investigación. Este instrumento está organizado en 44 ítems, los cuales valoran tres factores: actitudes hacia las relaciones sexuales, distintas maneras de practicar el sexo y expresión libre de la sexualidad, lo cuales se evaluaron mediante escala de Likert. El cuestionario, se aplicó de manera autoadministrada, a aquellos estudiantes que voluntariamente quisieron participar.
Para el procesamiento de la información se creó una base de datos en Excel y para el análisis estadístico se utilizó el paquete estadístico Statistical Package for Social Sciences (SPSS), versión 22.0. Se realizó análisis descriptivo de las variables y para establecer la relación entre las actitudes y las variables de interés se utilizó la prueba de Chi cuadrado.

También es importante anotar que en esta investigación se dio cumplimiento a lo establecidos en la Resolución 08430 del 1993 (10) del Ministerio de Salud de Colombia, que establece las normas técnicas y administrativas para la investigación con seres humanos. La propuesta investigativa, junto con sus aspectos éticos, fue evaluada y aprobada por el Comité Curricular del programa de enfermería de la universidad en estudio.

\section{OBJETIVOS}

\section{Objetivo general}

Describir las actitudes sexuales de los adolescentes matriculados en los programas presenciales diurnos de una universidad en Cúcuta, Colombia, durante el primer semestre de 2014.

\section{Objetivos específicos}

- Describir las actitudes hacia las relaciones sexuales que tienen los adolescentes.

- Describir las actitudes hacia las formas de practicar el sexo de los adolescentes.

- Describir las actitudes hacia la libre expresión de la sexualidad en los adolescentes.

- Establecer la relación entre las actitudes sexuales y algunas variables de interés.

\section{RESULTADOS Y DISCUSIÓN}

Los estudiantes que participaron en el estudio tenían, en promedio, 19 años de edad, predominando el género masculino con un 54\% y la mayoría pertenecía a los estratos 2 y 3 , con el $40 \%$ y $39 \%$ respectivamente. Respecto a la creencia religiosa, se observó una mayor proporción de estudiantes católicos con el $76 \%$; seguido de los protestantes representados por el 15\%, mientras que una baja proporción de estudiantes, un $6 \%$, se identifica como ateo. 
La alta proporción de estudiantes que afirma pertenecer a alguna organización religiosa no es proporcional con sus prácticas religiosas: el 32\% afirma que asiste al templo o a la iglesia algunas veces, mientras quienes lo hacen pocas veces equivale al $26 \%$. Los estudiantes provienen de familias de composición nuclear en un 55\%, seguido por aquellos de familias monoparentales con el $17 \%$ y extensas con $15 \%$. Respecto a la orientación sexual, predominó la heterosexual con un $82,9 \%$ y, en menor proporción, se encuentra la orientación bisexual y homosexual con un $6.9 \%$.

Figura 1. Actitud sexual en adolescentes universitarios.

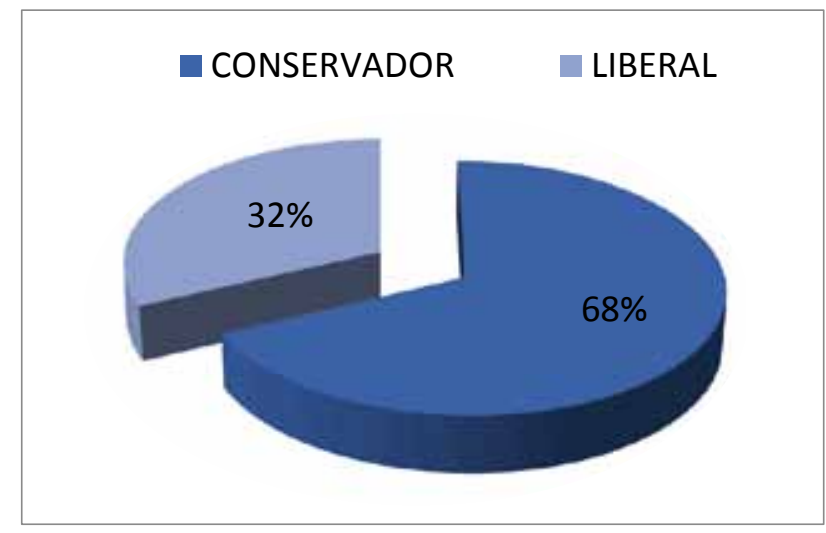

Fuente: Cuestionario Actitudes Sexuales (AS), 2005.

$\mathrm{Al}$ analizar los resultados obtenidos en los 40 ítems que evaluaron las actitudes sexuales, se encontró predominio de actitudes liberales en un $68 \%$, mientras que el porcentaje restante de estudiantes se mostró con actitud conservadora hacia la sexualidad.

Figura 2. Actitudes frente a las relaciones sexuales en adolescentes universitarios.

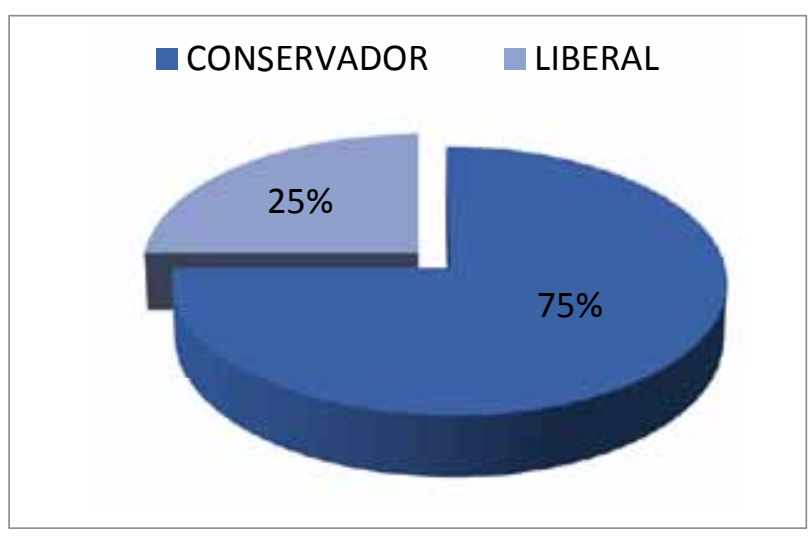

Fuente: Cuestionario Actitudes Sexuales (AS), 2005.
La actitud de los adolescentes hacia las relaciones sexuales, es predominantemente liberal en un $75 \%$, siendo baja la proporción de aquellos que expresaron tener unas actitudes conservadoras hacia la forma como se relacionan sexualmente con los demás.

Los adolescentes adoptaron posiciones liberales y permisivas en aspectos como la necesidad de sentir placer en el acto sexual con el 94,4\%, la importancia de experimentar diversas formas de tener relaciones sexuales con la pareja, $84 \%$, el sentimiento de bienestar al tener relaciones sexuales con frecuencia en un $80 \%$, aceptar que mantiene relaciones sexuales con $76 \%$, la aceptación del sexo oral dentro de las relaciones sexuales en un $72 \%$ y la importancia de este en la relación sexual con la pareja con el $62 \%$. Sin embargo, este último aspecto revela que, si bien los adolescentes aceptan la práctica del sexo oral en las relaciones en pareja y lo perciben como algo importante, no lo practican en el mismo grado en un $58 \%$.

Igualmente, la mayoría de los jóvenes estuvo de acuerdo con el sexo prematrimonial en un 58\% y de practicar sexo regularmente con el $57 \%$. Por otra parte, el ítem donde predominó la actitud conservadora con el $58 \%$, fue el de la expresión de sentimientos hacia la pareja por medio de las relaciones sexuales.

Figura 3. Actitudes de adolescentes universitarios hacia las distintas maneras de practicar el sexo.

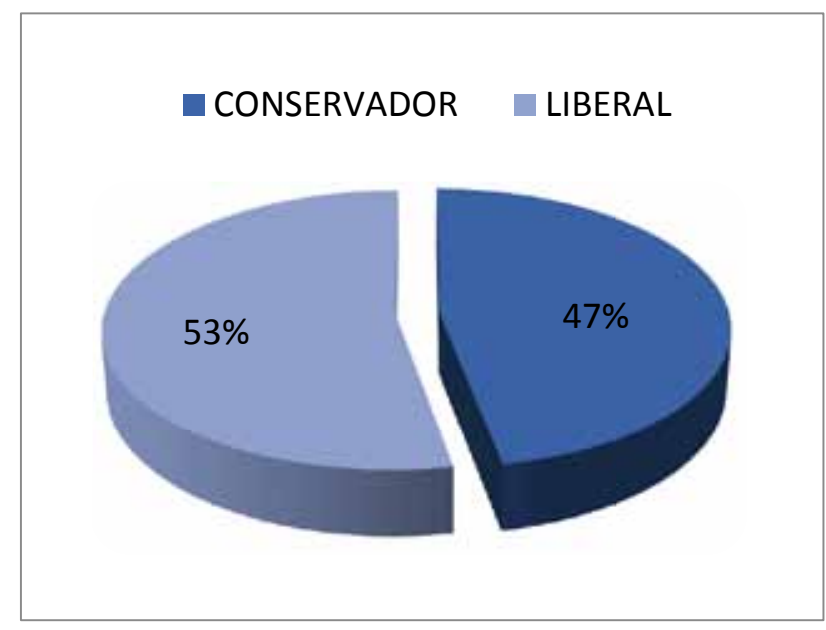

Fuente: Cuestionario Actitudes Sexuales (AS), 2005.

Contrario a las actitudes hacia la sexualidad y hacia las relaciones sexuales presentadas anteriormente, los adolescentes manifestaron en este ítem (Ver Figura 3) 
tener una actitud predominantemente conservadora sobre la forma de practicar el sexo, con un 53\%. Las actitudes conservadoras de los adolescentes se observaron al estar en desacuerdo con la validez del sexo anal en la vida sexual en pareja en un $45 \%$, la aceptación de su práctica con el $65 \%$ y el rechazo a masturbarse durante la relación sexual en un 59\%.

Sin embargo, el $64 \%$ considera que sí es válido practicar el sexo anal si la pareja lo acepta y el 53\% que la pareja se masturbe durante la relación sexual. Esta diferencia en los resultados demuestra que, si bien los adolescentes no aceptan desde su propio concepto algunas prácticas sexuales, esto cambia cuando se trata del placer de la pareja, lo que significa que complacer sexualmente al otro puede cambiar e influenciar el punto de vista del adolescente

Figura 4. Actitudes en adolescente universitarios hacia la libre expresión de la sexualidad.

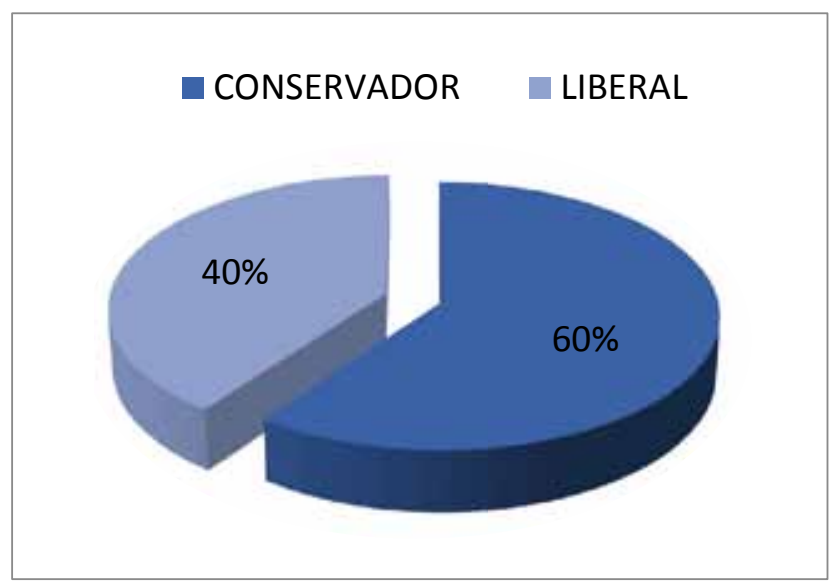

Fuente: Cuestionario Actitudes Sexuales (AS), 2005.

En el análisis de la actitud de los adolescentes hacia la libre expresión de la sexualidad, se encontró un porcentaje aún mayor de conservadores, $60 \%$, que de liberales, 40\%. Al analizar individualmente de los ítems que conforman este factor, se evidenció que las actitudes conservadoras prevalecen en la orientación sexual que manifiestan los estudiantes y en la práctica de la masturbación.

Por otra parte, los estudiantes se mostraron levemente más liberales cuando se preguntó si hablaban con su figura materna sobre sexo. El único ítem donde se observó una alta tendencia a las respuestas liberales fue en el de la importancia de conversar sobre sus sentimientos con la pareja antes del acto sexual.
Tabla 1. Relación entre variables de interés y actitud sexual.

\begin{tabular}{|c|c|c|c|c|c|}
\hline & & LIBERLL & CONSERVADOR & $\mathrm{x}$ & P \\
\hline \multirow[t]{2}{*}{ GENERO } & MASCTLINO & 176 & 25 & \multirow[t]{2}{*}{74,450} & \multirow[t]{2}{*}{0,000} \\
\hline & FESENINO & 80 & 94 & & \\
\hline \multirow[t]{2}{*}{ EDAD } & $16-18$ & 64 & $5 !$ & \multirow[t]{2}{*}{21,575} & \multirow[t]{2}{*}{$0, \infty$} \\
\hline & $19-20$ & 192 & 63 & & \\
\hline \multirow[t]{2}{*}{ ESTRATO } & 1.2 & III & 60 & \multirow[t]{2}{*}{5,173} & \multirow[t]{2}{*}{0,160} \\
\hline & 34 & 145 & 39 & & \\
\hline \multirow[t]{2}{*}{ SEMESTRE } & 16 & 132 & 83 & \multirow[t]{2}{*}{20,668} & \multirow[t]{2}{*}{0,00} \\
\hline & $6-10$ & 124 & 36 & & \\
\hline \multirow[t]{5}{*}{ FACLITAD } & INGENERLS & 9) & $4 !$ & \multirow[t]{5}{*}{3,713} & \multirow[t]{5}{*}{0,44} \\
\hline & EAPRESARALES & 102 & 44 & & \\
\hline & HLXLADADES & 30 & 15 & & \\
\hline & AGRLRLAS & 26 & If & & \\
\hline & SALTD & 8 & I & & \\
\hline \multirow[t]{4}{*}{ RELIGIOX } & CATOLICO & 205 & 80 & \multirow[t]{4}{*}{12,024} & \multirow[t]{4}{*}{0,007} \\
\hline & PROTESTANTE & 29 & 29 & & \\
\hline & AGIOSTICO & 9 & 2 & & \\
\hline & ATEO & 13 & 8 & & \\
\hline \multirow[t]{6}{*}{ TIPOEAIILA } & NUCZEAR & 136 & 72 & \multirow[t]{6}{*}{4,865} & \multirow[t]{6}{*}{0,430} \\
\hline & EXTENSA & 45 & 16 & & \\
\hline & MONOPARENTERAL. & 46 & 17 & & \\
\hline & AMPLIDA & I & 1 & & \\
\hline & STMLLTAEA & 2 & 3 & & \\
\hline & PADRASTRLL & 31 & 10 & & \\
\hline \multirow{5}{*}{$\begin{array}{l}\text { COMPOSICTON } \\
\text { FAMILAR }\end{array}$} & CASADOS & 125 & 72 & \multirow[t]{5}{*}{7,324} & \multirow[t]{5}{*}{0,120} \\
\hline & DIVORCLADOS & 83 & 32 & & \\
\hline & $\begin{array}{l}\text { PADRE O JADRE } \\
\text { IITDO }\end{array}$ & 23 & II & & \\
\hline & $\begin{array}{l}\text { AMBOS } \\
\text { FALLECTDOS }\end{array}$ & 1 & 0 & & \\
\hline & TNONLIBRE & 24 & 4 & & \\
\hline \multirow{3}{*}{$\begin{array}{l}\text { POSTLRA } \\
\text { RELIGIOSA }\end{array}$} & CREYENTE & 234 & 109 & 1,325 & 0,516 \\
\hline & AGNOSIICO & 9 & 2 & & \\
\hline & ATEO & 13 & 8 & & \\
\hline ASISTENCIA & SIEAPRE & 22 & 20 & 18,923 & $0, \infty 01$ \\
\hline AL. TEXPLO & FRECTENTEMENTE & 50 & 36 & & \\
\hline & AIECES & $\$ 5$ & 25 & & \\
\hline & POCO & 6 & 33 & & \\
\hline & IECA & 25 & 5 & & \\
\hline ORIENTACON & HETEROSEXTAL & 213 & 98 & 12,920 & 0,005 \\
\hline SEXUAL & GAY & 2 & 7 & & \\
\hline & LESBLAXI & 2 & 6 & & \\
\hline & BISEXTAL & 24 & 2 & & \\
\hline & INDECTSO & 10 & II & & \\
\hline
\end{tabular}

Fuente: Cuestionario Actitudes Sexuales (AS), 2005.

Al realizar la evaluación de las variables de interés se encontró que la actitud liberal tiene una asociación estadísticamente significativa con el género masculino, ser bisexual, tener entre 18 y 19 años, estar cursando entre 6 y 10 semestre, ser agnóstico o ateo y asistir pocas veces o nunca al templo o la iglesia. Igualmente, no se encontró asociación estadística 
entre la actitud liberal y el estrato socioeconómico, la facultad en la que está matriculado, el tipo de familia, la composición familiar o la postura religiosa.

El predominio de las actitudes sexuales liberales observadas es consistente con lo reportado por Libreros et al. (2) y Lameiras (11), quienes observaron un predomino en los adolescentes de la actitud permisiva, liberal y favorable hacia el ejercicio de la sexualidad, aceptando la masturbación, el contenido erótico y sexual, y la homosexualidad ajena. Si bien se mantienen actitudes liberales, se destaca que los adolescentes conservan actitudes conservadoras respecto a la promiscuidad sexual y al acceso o participación en la prostitución.

Aguirre (12) afirma que la actitud liberal (no sólo hacia el sexo sino también hacia múltiples aspectos de la vida), es inherente a la etapa de la adolescencia debido a la búsqueda implacable de la propia identidad y la libertad e independencia.

Estas actitudes se deben a la necesidad de explorar el mundo, la adquisición de independencia en la etapa universitaria, la baja noción del riesgo y la presión ejercida por sus grupos de iguales, lo que los lleva a vivir su sexualidad sin inhibiciones (5). Tal y como lo menciona González et al. (13), las actitudes que toman los adolescentes son influenciadas por múltiples factores, entre los que se encuentran la cultura y las relaciones interpersonales. Estas actitudes, pueden llegar a transformarse en conductas de riesgo tales como el ejercicio prematuro de la sexualidad, por la poca autorregulación que tienen los adolescentes (14$15)$.

La preeminencia, en los estudiantes universitarios, de actitudes liberales frente a las relaciones sexuales, observada en esta investigación, concuerda con lo descrito en el Segundo Informe Nacional de Juventud, donde se evidencia que los jóvenes tienen relaciones sexuales principalmente por deseo, más que por amor, cambio que se ha venido presentando en las últimas décadas (15).

Mate y Acarín (16) encontraron que, para los adolescentes, la edad ideal para iniciar relaciones sexuales oscila entre los 16 y 19 años; además, mostraron que la mayoría representada por el $80 \%$, inició relaciones sexuales por el deseo de experimentar placer o por una aventura, mientras que apenas un 14\% afirmó haber iniciado su vida sexual por amor hacia su pareja.

Mate y Acarín (16) también revelaron que los adolescentes retrasarían el inicio de la vida sexual activa por razones como la importancia que le dan al estudio, encontrar a la persona con la que se sintieran totalmente a gusto y esperar llegar vírgenes al matrimonio, en un $17 \%$. Las motivaciones para iniciar relaciones sexuales en los adolescentes están más vinculadas al sexo recreativo que al sexo afectivo, dándole prioridad a la búsqueda del placer antes que al establecimiento de relaciones sólidas basadas en el campo afectivo.

Campos (17) afirma que las relaciones en la adolescencia tienen un fin principalmente de satisfacción física antes que sentimental, debido a la carga hormonal a la que están expuestos durante esta etapa de su desarrollo, especialmente por la testosterona en los hombres. Al respecto Benítez y Rueda (18) presentan otra explicación ellos encontraron que el inicio y disfrute de las relaciones sexuales en los adolescentes está influenciado por factores como el acceso a material pornográfico.

Los estudiantes que participaron en el estudio manifestaron tener una actitud predominantemente conservadora sobre la forma de practicar el sexo, en contraposición a las actitudes liberales hacia la sexualidad y hacia las relaciones sexuales que se evidencian en los párrafos anteriores.

Esta diferencia en los resultados demuestra que, a pesar de que los adolescentes no aceptan desde su propio concepto algunas prácticas sexuales, esta perspectiva cambia cuando se trata del placer de la pareja (19). Noboa y Serrano (20), mostraron que las adolescentes eran más permisivas si la pareja lo pedía, ya que para ellas no es correcto negarle el placer a la pareja.

El predomino de la actitud conservadora hacia la libre expresión de la sexualidad también se observó en el estudio de García (9), donde las actitudes hacia la expresión libre de la sexualidad mostraron la menor proporción de liberales respecto a los otros factores. Evidentemente, la mayoría de los adolescentes son reservados cuando se trata de hablar de su sexualidad, de compartir sus sentimientos con su pareja y de expresar sus orientaciones sexuales. 
Chávez et al. (21), encontraron en su estudio que los adolescentes manifestaban un fuerte rechazo ante la homosexualidad, tildando de anormal este tipo de relación y catalogándola como un tipo de enfermedad mental. Sin embargo, un $13 \%$ de los adolescentes que dijeron ser heterosexuales manifestó haber mantenido, en alguna ocasión, relaciones sexuales con parejas de su mismo sexo.

Respecto a la comunicación sobre sexualidad con la figura materna de la familia, los resultados concuerdan con el estudio de Bautista (22), donde la principal fuente de información sobre sexualidad para los adolescentes es la familia y los adolescentes consideran que la información que reciben es confiable y la tienen en cuenta al momento de tomar decisiones en materia de sexualidad.

Wilhelm (23), halló en su estudio que los adolescentes varones admiten con frecuencia una falta de comunicación con su figura paterna $\mathrm{y}$, en muchos casos, cuando existe comunicación, los mensajes promueven el sexo irresponsable, alientan la actividad sexual temprana y refuerzan los conceptos de machismo.

Se considera que existen variables que condicionan las actitudes de los adolescentes frente a su sexualidad. Esto refleja la teoría de Dorothy Johnson, de acuerdo con Cortes (24), en la cual se plantea que el ser humano es un sistema conductual en el que aspectos externos como su entorno y algunos factores sociodemográficos, familiares, de género, la religión que profesan y la edad, entre otros, orientan o condicionan su manera de actuar, especialmente si se trata de comportarse de forma liberal o conservadora respecto a la sexualidad.

Los resultados indican que los hombres tienen una actitud más liberal que las mujeres, fenómeno similar a lo encontrado por Ochaita y Espinoza (25), quienes observaron que las mujeres son más conservadoras en comparación con los hombres. Igualmente, Weiss y Vargas (26), observaron que los hombres tienen más probabilidades de haber tenido relaciones sexuales y suelen informar sobre el comienzo de la actividad sexual a una edad más temprana que las mujeres (27).

Los adolescentes de mayor edady los que se encuentran en semestres más avanzados presentaron conductas más liberales. Estos hallazgos son consistentes con lo evidenciado en el estudio realizado por Diéguez et al. (28), quienes observaron que la puntación media en la escala se incrementa con la edad, es decir, las personas más conservadoras son las de menor edad y las de los primeros semestres.

En relación con las creencias religiosas, se observó que las personas agnósticas o ateas y aquellas que asisten pocas veces o nunca al templo presentan actitudes liberales con respecto a la sexualidad, las personas que no profesan ninguna religión son más liberales, mientras que aquellos que reconocen y profesan alguna religión tienden a mantener actitudes conservadoras, mostrando rechazo hacia las practicas liberales (29). Moral et al. (30) evidenciaron que aquellas personas que profesaban una religión y asistían frecuentemente al culto mostraban actitudes más conservadoras, rechazando las prácticas liberales por vergüenza hacia todo lo que encierra la sexualidad.

La actitud sexual liberal que se observó, en este estudio, en los estudiantes que se identificaban como bisexuales, es similar a lo reportado por García (10), quien encontró una relación estadísticamente significativa entre ser hombre bisexual y tener actitudes sexuales más liberales, seguido de los heterosexuales y los indecisos.

\section{CONCLUSIONES}

Los adolescentes universitarios expresan un liberalismo sexual que es más discursivo que practico, observándose actitudes liberales pero conductas más conservadoras. En cada uno de los aspectos evaluados, las frecuencias fueron disminuyendo, pasando del liberalismo en las actitudes respecto a las relaciones sexuales hacia una postura conservadora en relación con las actitudes frente a las prácticas y la expresión libre de la sexualidad.

La mayoría de los estudiantes encuestados mantiene actitudes liberales sobre la sexualidad. Se destacan aspectos como la importancia que los adolescentes le dan al sexo prematrimonial, asunto que demuestra que la virginidad hasta el matrimonio no es una cuestión de importancia para ellos y que conocerse íntimamente con la pareja, antes de llegar al matrimonio, es visto de forma aceptable. Igualmente, es importante para ellos disfrutar del placer sexual, lo que puede influenciar las opiniones de los adolescentes cuando se trata de conseguir que ellos mismos o su pareja logren satisfacción durante la relación sexual 
Los adolescentes se muestran más conservadores en relación con las vivencias de la sexualidad. En este factor predominaron las actitudes no permisivas o conservadoras hacia las prácticas diferentes a las relaciones sexuales vaginales tradicionales, puesto que los adolescentes no las consideran aceptables y afirman no practicarlas; además, reiteran que el coito vaginal es el único que debe aceptarse durante el acto sexual. Sin embargo, sus posturas cambian si se trata de complacer a su pareja, ya que ven de forma positiva estas prácticas si su compañero sexual las acepta, lo cual puede indicar que prima el deseo de satisfacer a la pareja antes que su propia opinión.

También se encontró que existe relación estadísticamente significativa entre poseer actitudes liberales y pertenecer al género masculino, tener edad superior o igual a 19 años, pertenecer a los semestres superiores, ser agnóstico o ateo, asistir poco o nunca al templo y ser bisexual. Por otra parte, aquellas variables estudiadas que no mostraron asociación estadística con poseer o no actitudes liberales hacia la sexualidad, son: el estrato socioeconómico, la facultad en la que estudian los adolescentes, el tipo de familia, la composición familiar y la postura religiosa.

La sexualidad en los jóvenes universitarios puede generar situaciones de riesgo y tener graves implicaciones para la salud sexual y la calidad de vida; por ello, resulta imperativo incluir dentro de los programas de bienestar universitario estrategias de promoción y prevención tendientes a fortalecer una sexualidad sana, libre, responsable y placentera en los estudiantes. 


\section{REFERENCIAS BIBLIOGRÁFICAS}

1. Navarro Y, Sánchez JC. Comportamientos y actitudes sexuales en adolescentes y jóvenes. Revista Hispanoamericana de Sexología. [Internet]. 2004 [consultado 13 de octubre de 2013]; 3 (1): 4-10. Disponible en: https://es.scribd.com/ doc/22158992/Comportamientos-y-Actitudes-Sexuales-en-Adolescentes

2. Libreros L, Fuentes L, Pérez A. Conocimientos, actitudes y prácticas sobre sexualidad de los adolescentes en una unidad educativa. Respin [Internet]. 2008 [consultado 08 de mayo de 2014]; 9(4). Disponible en: http://www.respyn. uanl.mx/ix/4/articulos/articulo_actitudes_sexualidad.htm

3. Programa Conjunto de las Naciones Unidas sobre el VIH/sida - ONUSIDA. Informe sobre la epidemia mundial de sida. Resumen de orientación. [Internet]. Ginebra: Naciones Unidas sobre el VIH/Sida; 2008 [consultado 13 de octubre de 2013]. Disponible en: http://data.unaids.org/pub/globalreport/2008/jc1511_gr08_executivesummary_ es.pdf

4. Milhausen R, Herold E. Does the sexual double standard still exist "Perceptions of university women"? The Journal of Sex Research [Internet]. 1999. [consultado 18 de marzo de 2014]; 36(4): 361-368. Disponible en: http://www. tandfonline.com/doi/abs/10.1080/00224499909552008\#.VDG60v15NqU

5. Hatano Y. Changes in the sexual activities of Japanese youth. Journal of Sex Education \& Therapy [Internet]. 2004 [consultado 18 de marzo de 2014]; 17 (1): 1-14. Disponible en: http://psycnet.apa.org/psycinfo/1991-27092-001

6. Universidad Francisco de Paula Santander. Boletín Estadístico I Semestre 2014. Unidad de Información y Estadística. [Internet]. Cúcuta: Oficina de Planeación - UFPS; 2014. [consultado 08 de mayo de 2014]. Disponible en: http:// planeacion.ufps.edu.co:9191/Planeacion/web/index.ufps

7. República de Colombia - Ministerio de la Protección Social. Situación de la epidemia de VIH/sida en Colombia. [Internet]. Bogotá: Oficina Asesora de Comunicaciones; 2012. [consultado 13 de octubre de 2013]. Disponible en: http://www.minsalud.gov.co/Documentos\%20y\%20Publicaciones/BOLETIN\%20 EPIDEMIOLOGICO $\% 20$ VIH\%201983-2012.pdf

8. Asociación Probienestar de la Familia Colombiana - PROFAMILIA. Salud sexual y reproductiva en Colombia. Encuesta Nacional de Demografía y Salud 2010. [Internet]. Bogotá: PROFAMILIA; 2010 [consultado 13 de octubre de 2013]. Disponible en: http://www.profamilia.org.co/ encuestas/Profamilia/Profamilia/index.php?option=com_co ntent\&view $=$ article $\&$ id $=62 \&$ Itemid $=9$

9. García-Belaúnde Velarde VA. Actitudes sexuales de adolescentes y jóvenes universitarios. [Internet]. 2005 [consultado 13 de octubre de 2013]. Disponible en: http://www.academia.edu/1314849/Actitudes_sexuales_en_ adolescentes_y_jovenes_universitarios

10. República de Colombia. Ministerio de Salud. Resolución 08430 del 1993, 4 de octubre, por la cual se establecen las normas científicas, técnicas y administrativas para la investigación en salud. Santafé de Bogotá D.C.: Ministerio de Salud; 1993.

11. Lameiras-Fernandez M. El estudio de la sexualidad en jóvenes españoles. InterAÇÃO, Curitiba; [Internet]. 1998 [consultado 13 de octubre de 2013]; 2:133-161. Disponible en: http://ojs.c3sl.ufpr.br/ojs/index.php/psicologia/ article/viewFile/7651/5457

12. Aguirre A. Psicología de la adolescencia. España: Editorial Boixareu Universitaria; 1994.

13. González E, Molina T, Montero A; Martínez V Molina R. Factores asociados a la continuidad del uso anticonceptivo en adolescentes solteras nulíparas. Rev. Méd. Chile 2009; 137(9):1187-1192.

14. Uribe A, Orcasita L. Conductas sexuales de riesgo en estudiantes universitarios de la ciudad de Cali-Colombia. Revista Virtual Universidad Católica del Norte [Internet]. 2009 may- agos [consultado 2 de mayo del 2014]; (27):131. Disponible en: http://revistavirtual.ucn.edu.co/index.php/RevistaUCN/article/view/99

15. Filardo V,Cabrera M, Aguiar S. Encuesta Nacional de Adolescencia y Juventud. [Internet]. Montevideo; Instituto Nacional de la Juventud; 2010 [consultado 19 marzo 2014]. Disponible en: http://www.inju.gub.uy/innovaportal/ file/9798/1/enaj_segundo_informe_cap1y2.pdf

16. Maté $\mathrm{C}$, Acarín N. las relaciones sexuales, encuesta a los estudiantes de la Universidad Pomepu Fabra, Barcelona (2027 años). SUMMA Psicológica UST. [Internet]. 2010 [consultado 19 de marzo de 2014]; 7 (2):93 - 108. Disponible en: http://dialnet.unirioja.es/descarga/articulo/3423964.pdf

17. Campos A. Relaciones sexuales en adolescentes colombianos y las implicaciones para la salud pública: una revisión de la prevalencia y algunas variables asociadas. MedUNAB [Internet]. 2009 [consultado 19 de marzo de 2014]; 12(2):86-90. Disponible en: http://revistas.unab.edu.co/index.php?journal= medunab\&page=article\&op=viewArticl e\&path $\% 5 B \% 5 \mathrm{D}=35$

18. Benítez L, Rueda C. Factores determinantes para la iniciación sexual y prácticas sexuales en jóvenes universitarios en Cali, Colombia. Salud Libre. [Internet]. 2007 [consultado 18 de mayo de 2014]; 2 (2): 90-110. Disponible en: http://www.unilibrecali.edu.co/Revista_Colombiana_Salud_Libre/vol_22/ factores\%20determinantes $\% 20$ para $\% 20$ la\%20iniciacion\%20sexual.pdf 
19. Cunningham I, Rodríguez-Sánchez M. Bibliografía sobre los aspectos epidemiológicos, sociales, legales y éticos Del SIDA. [Internet]. San Juan, P.R.: Instituto de Estudios del Caribe: 2010 [consultado 19 de mayo del 2014]. Disponible en:http://books.google.com.co/books/about/Bibliograf\%C3\%ADa_sobre_los_aspectos_epidemi.html?id/

20. Noboa-Ortega, Serrano-García I. Autoeficacia en la negociación sexual: retos para la prevención de VIH/SIDA en mujeres puertorriquenas. Rev. Latinoam. Psicol [Internet]. 2006 [consultado 19 de febrero de 2014]; 38(1): 21-43. Disponible en:http://pepsic.bvsalud.org/scielo.php?script=sci_arttext\&pid= S0120-05342006000100002\&lng=pt\& nrm=iso $>$. ISSN $0120-0534$

21. Chávez M, Petrzelová J, Zapata J. Actitudes respecto a la sexualidad en estudiantes universitarios Enseñanza e Investigación en Psicología [Internet]. 2009 [consultado 13 de octubre de 2013]; 14(1):137-151. Disponible en: $\mathrm{http}: / / \mathrm{www}$. redalyc.org/articulo.oa?id=2921411

22. Bautista L. Conocimientos, actitudes y opiniones de las y los universitarios oaxaqueños respecto a su sexualidad y la importancia de la educación sexual en el nivel de educación superior. Centro regional de investigación en psicología. [Internet]. 2008 [consultado 13 de octubre de 2013]; 2 (1):71-84. Disponible en: http://www.imbiomed.com.mx/1/1/ articulos.php?method $=$ showDetail\&id articulo $=51596 \&$ id seccion $=3247 \&$ id ejemplar $=5224 \&$ id revista $=170$

23. Wilhelm R. La función del orgasmo. $3^{\bar{a}}$ ed. [Internet]. New York: Editorial Paìdós Ibérica; 2010. [consultado 19 de mayo de 2014]. Disponible en: http://mastor.cl/blog/wp-content/uploads/2014/09/La-funci\%C3\%B3n-del-orgasmo.W-Reich.pdf

24. Cortes JE. La resiliencia: una mirada desde la enfermería. [Internet]. 2010 [consultado 10 de noviembre de 2014]; 16 (3): 27 - 32. Disponible en: http://www.scielo.cl/pdf/cienf/v16n3/art_04.pdf

25. Ochaita E, Espinosa A. Las prácticas sexuales de los adolescentes y jóvenes españoles. Estudios de juventud. [Internet]. 2003 [consultado 19 de febrero del 2014]; 63:49-62. Disponible en: http://www.injuve.es/sites/default/ files/07\%20PRACTICAS\%20SEXUALES63.pdf

26. Weiss $\mathrm{S}$, Vargas E. Conducta sexual y reproductiva de los adolescentes en América Latina. Revista Avances en Psicología Clínica Latinoamericana. 2010; 8:11- 41.

27. Shortt JW, Capaldi DM, Dishion TJ, Bank L, Owen LD. The Role of Adolescent Friends, Romantic Partners, and Siblings in the Emergence of the Adult Antisocial Lifestyle. J Fam Psychol. [Internet]. 2003 Dec [consultado 18 de marzo de 2014]; 17(4):521-33. Disponible en: http://www.ncbi.nlm.nih.gov/pubmed/14640802

28. Diéguez-Ruibal JL, López-Castedo A, Sueiro-Domínguez E, López-Sánchez F. Propiedades psicométricas de la escala de actitudes hacia la sexualidad (ATSS) ampliada. C. Med. Psicosom [Internet]. 2005 [consultado 5 de mayo de 2014]; 74:46-56. Disponible en: http://www.uned.es/master-psicologia-deporte/sp/pdf/psi_actitudes.pdf

29. Moral-de la Rubia J. Religión, significados y actitudes hacia la sexualidad: un enfoque psicosocial. Revista colombiana de psicología. [Internet]. 2010 [consultado 5 de mayo de 2014]; 19(1): 45-59. Disponible en: http:// www.revistas.unal.edu.co/index.php/psicologia/article/view/974/36671

30. Moral J, Álvarez L, Ibarra L. Religión, emociones y conducta sexual en jóvenes universitarios. Investigaciones en psicología social, de la personalidad y la salud. [Internet]. México: CUMEX; 2004 [consultado 5 de mayo de 2014] Disponible en: http://bvs.sld.cu/revistas/mciego/docencia/psicologia_salud/paginas/pagina01/Tema3.html_ 